\title{
Quels profils pour les métiers de la communication d'entreprise?
}

Jean-Luc Michel

\section{(2) OpenEdition}

1 Journals

Édition électronique

URL : http://journals.openedition.org/communicationorganisation/2076

DOI : 10.4000/communicationorganisation.2076

ISSN : $1775-3546$

Éditeur

Presses universitaires de Bordeaux

Édition imprimée

Date de publication : 1 novembre 1998

ISSN : 1168-5549

\section{Référence électronique}

Jean-Luc Michel, «Quels profils pour les métiers de la communication d'entreprise ? ", Communication et organisation [En ligne], 14 | 1998, mis en ligne le 26 mars 2012, consulté le 19 avril 2019. URL:

http://journals.openedition.org/communicationorganisation/2076 ; DOI : 10.4000/

communicationorganisation.2076

Ce document a été généré automatiquement le 19 avril 2019.

(c) Presses universitaires de Bordeaux 


\title{
Quels profils pour les métiers de la communication d'entreprise?
}

\author{
Jean-Luc Michel
}

1 Les journées d'études de la SFSIC et les publications des professionnels de l'information et de la communication ont établi à plusieurs reprises la pérennité des métiers et des fonctions de la communication d'entreprise ou d'organisation. On observe encore deux « marchés », un « marché de conquête » (ou de " premier équipement ») qui concerne des PME/PMI, des administrations ou des petites villes qui créent des postes de communication sur des profils de bi ou de polycompétence, et à l'opposé, un « marché de renouvellement » où des services de communication anciens embauchent des diplômés pour des tâches précises et de plus en plus diversifiées (responsable du mécénat ou du parrainage, chargé de communication de crise, attaché de communication financière, etc.). Cet article s'appuie sur des travaux de recherche visant à repérer et modéliser quelques "profils types» de communicants à partir d'un répertoire de toutes les fonctions liées à la communication.

\section{Une pérennité assurée}

2 Les enquêtes socioprofessionnelles l'attestent depuis plusieurs années : les fonctions de communication ne font plus l'objet de débats dans les entreprises et les administrations ; on ne peut se passer de professionnels de la communication ${ }^{1}$. Les recrutements n'ont pas cessé, même au cœur de la crise (1994 à 1996) et repartent fortement en 1997/1998², même si d'autres phénomènes, qui ne seront pas analysés ici peuvent devenir préoccupants, comme la baisse continue des rémunérations et l'augmentation des tâches à accomplir, tant en quantité (horaires de plus en plus souples, "disponibilité " et «mobilité » de plus en plus grandes), qu'en qualité (« adaptabilité » et polycompétence de plus en plus requises, sans pour autant que le statut de cadre soit garanti, voire une absence totale de statut comme dans la fonction publique territoriale). Le vieux débat sur le positionnement des fonctions de communication interne par rapport aux directions des ressources humaines est pratiquement clos au profit de la communication qui a su 
montrer son dynamisme et son efficacité. Nous conseillons toujours à nos étudiants de revendiquer un rattachement direct auprès de la direction générale, c'est là qu'ils sont le plus libre.

Même de nouveaux modes de management comme le travail à distance ne semblent pas atteindre le statut ni les responsabilités des communicants, d'autant que ceux-ci (on devrait plutôt dire celles-ci car la féminisation est très forte, entre 70 et $80 \%$ ) sont de mieux en mieux formés ${ }^{3}$.

4 Après la grande conquête des années 80 , nous sommes dans un contexte de diversification, le «marché » des recrutements, à l'instar de beaucoup d'autres, se segmente de plus en plus finement, mais sans que l'on sache bien (les employeurs encore moins que les autres) quels métiers et quelles compétences doivent être proposés par le système de formation.

5 La recherche qui a donné lieu à cet article s'était fixé comme premier objectif d'y voir plus clair, ou en termes plus sérieux de concevoir un référentiel des fonctions de base de la communication d'entreprise 4 à partir duquel on pourrait décrire finement les métiers et prévoir leurs évolutions.

\section{Des fonctions aux métiers}

6 Après une vision plutôt globale mais imprécise dans les années 70 (publicité, relations publiques, directeur de la communication ou « dircom »5), on a observé une spécialisation croissante des tâches et des fonctions sans que pour autant il soit possible de définir précisément des «métiers » de la communication d'entreprise ${ }^{6}$. L'alternative théorique est simple : soit on part de métiers que l'on observe sur le terrain, soit on décrit les tâches les plus fines et on remonte aux métiers. C'est évidemment la démarche « atomiste » que nous avons privilégiée, mais en la corrélant d'une forte vision systémique. Le repérage de «descripteurs" très fins, voire de «micro-descripteurs» de la communication va répondre à trois objectifs emboîtés :

7 1. Une connaissance approfondie et facilement évolutive des fonctions (construite à partir d'assemblages de descripteurs).

8 2. Une description fidèle, précise et dynamique des métiers à partir des fonctions.

9 3. Une modélisation permettant des hiérarchisations aisées des tâches et une prospective fiable des évolutions.

10 Mais la présente méthode va encore plus loin que la description, même dynamique, des fonctions et des métiers. Elle peut permettre aux employeurs de spécifier des profils de tâches et/ou de responsabilités tout en donnant le moyen de rendre plus fiables les procédures de recrutement. Comme dans toutes les activités récentes, il est en effet difficile pour un employeur de connaître le niveau de compétences requis par telle ou telle tâche dont il a besoin. Les enseignants de communication le savent bien: des demandes de stages de niveau Bac +2 sont souvent présentées à des étudiants de maîtrise ou de DESS et il est parfois difficile aux étudiants de faire comprendre à un employeur, même de bonne volonté, ce qui est de leur niveau de connaissance et de pratique professionnelle. Et si la poly-compétence est le plus souvent de rigueur, il ne saurait être question de tout mélanger sous prétexte d'adaptabilité servile ; mieux vaut un référentiel 
précis, au carrefour des observations de terrain et des évolutions les plus récentes, pour construire à terme une sociologie des métiers.

\section{Une vision systématique indispensable}

11 Avant de dresser une liste des descripteurs des fonctions, il est nécessaire de rappeler brièvement les principales tâches à remplir par la communication, des plus anciennes et mieux connues aux plus récentes. L'efficacité descriptive et l'observation attentive du terrain imposent une vision systémique seule capable de réunir dynamiquement - et dialectiquement - les composantes internes et externes de la communication (cette approche est souvent qualifiée de "globale» et stratégique ${ }^{7}$ ) - Le tableau ci dessous illustre les principaux aspects internes ou externes de la plupart des tâches classiques. Ce sera notre point de départ.

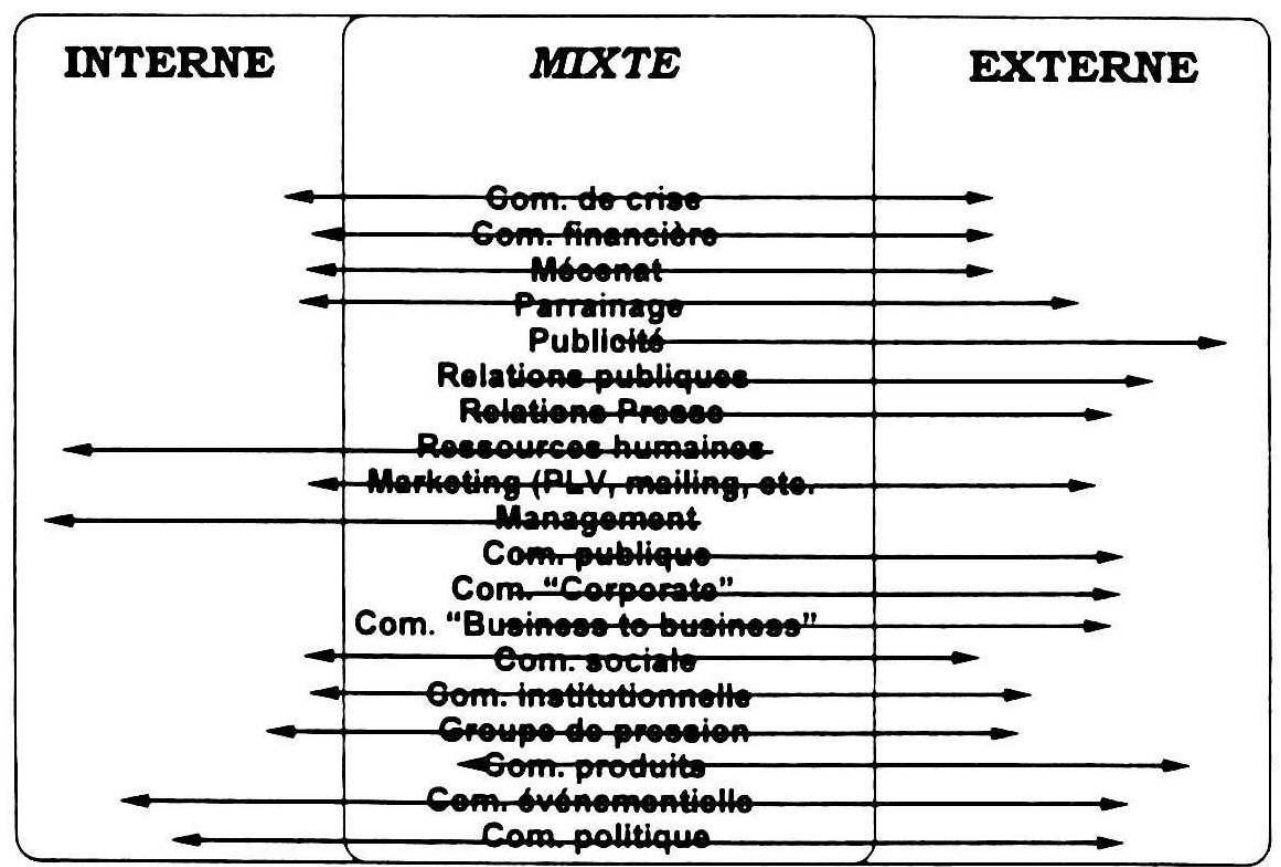

Figure 1 : Quelques unes des principales composantes de la communication d'entreprise

Cette cartographie, non commentée par manque de place, sépare l'interne, l'externe et une zone intermédiaire dénommée " mixte ». Chaque entreprise ou organisation possède les trois dans des proportions variables en fonction de ses caractéristiques propres, de même que chaque communicant jouit de compétences plus affirmées dans un domaine ou dans un autre. La bonne adéquation consistant naturellement pour chaque entreprise à trouver un collaborateur correspondant à son spectre d'activités privilégiées.

\section{Les descripteurs}

La grille ci dessous a été construite en trois parties ; la première présente les fonctions concernant plutôt l'interne, la seconde plutôt l'externe et la troisième ce qui est de tendance mixte. La première version, élaborée en 1995, comprenait une centaine d'items, la version 1998 en comporte 139. Le plus important à signaler concerne son aspect 
dynamique et auto-évaluatif. La grille a été saisie sur un tableur avec un masque de saisie pour chacun des items; les étudiants peuvent s'auto-évaluer en se notant sur chacun d'eux à l'aide d'une échelle graduée de 1 à 5 , les totalisations s'effectuent automatiquement, de sorte qu'en fin de saisie, l'intéressé connaît instantanément son «score » et surtout son profil personnel, indiqué par une trentaine de grandes fonctions. Il est évidemment possible de remplacer l'auto-évaluation par une évaluation extérieure (éventuellement normative...) ou d'employer la grille «à l'envers", par exemple pour déterminer quel profil correspondra à telle famille de tâches.

\section{Communication externe classique}

5 Communication produit

11 Suivi de l'opération qualité

15 Négociation

18 Direction et suivi de projets

19 Achat et gestion d'espace

20 Relations publiques

23 Suivi de l'opération RP

24 Relations Presse

26 PLV

27 « Mailing »

28 «Phoning ${ }^{\circ} »$

29 Presse d'entreprise externe
1 Définition d'une stratégie de communication

2 Détermination des objectifs d'une campagne publicitaire

3 Organisation du choix de la sous-traitance publicitaire

4 Suivi de l'opération publicitaire

6 Communication institutionnelle

7 Communication événementielle

8 Conception/Gestion/Évaluation d'une opération de mécénat

9 Conception/Gestion/Évaluation d'une opération de parrainage

10 Détermination des objectifs externes d'une campagne de qualité

12 Communication de notoriété

13 Contacts avec agences conseil en communication

14 Compétences en langues étrangères pour traduction

16 Cahiers des charges - Budget

17 Sélection des soumissionnaires

21 Déterminer les objectifs d'une opération RP

22 Organiser le droit de la sous-traitance RP

25 Liaisons avec direction du Marketing 


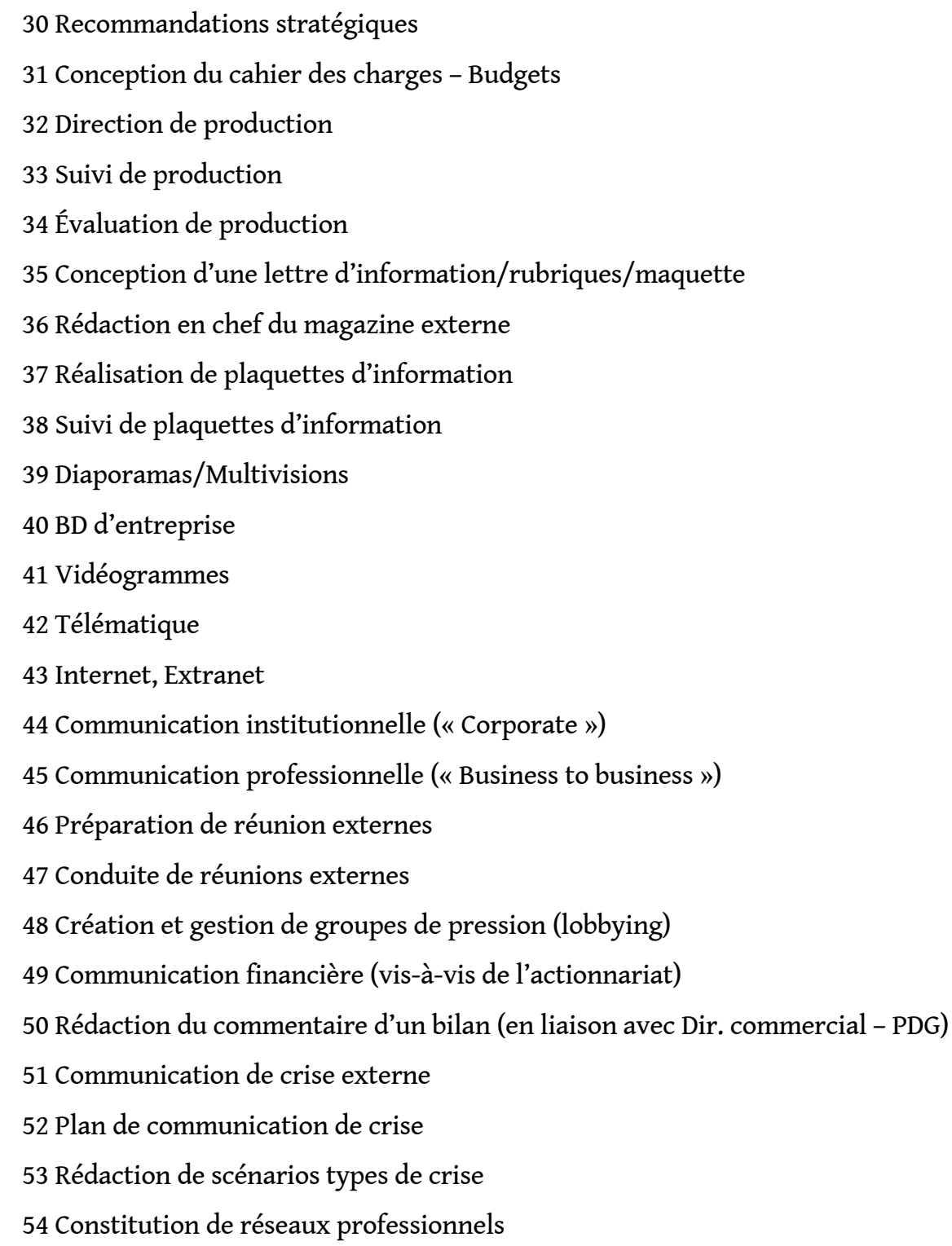

\section{Communication stratégique et globale}

1 Analyse stratégique

2 Analyse de l'identité

3 Analyse de l'image (voulue, perçue, vécue)

4 Analyse des enjeux

5 Audit

6 Marketing interne

7 Bilan social

8 Diagnostic de situation

9 Préconisations/Recommandations stratégiques

10 Conseils au comité de direction 
11 « Coaching » des dirigeants

12 Conseil interne - sous-traitance interne

13 Objectifs du conseil externalisé

14 Choix et gestion de la sous-traitance

15 Plan de communication

16 Cahier des charges

17 Budget global - budget analytique

18 Suivi de projets

19 Communication politique

20 Communication publique

21 Gestion des délégations de pouvoir

22 Évaluation de la communication stratégique et globale

\section{Les principales fonctions}

Les 139 items précédents se combinent entre eux pour donner, dans la version 1998 de la recherche 32 fonctions principales. La souplesse du système est telle qu'il est évidemment possible qu'un descripteur soit employé dans deux ou plusieurs fonctions distinctes; ce qui compte, c'est la combinatoire, laquelle renferme du dynamisme et de l'évolutivité8.

\section{2 fonctions principales}

Écrits internes

Écrits externes

Audiovisuel/Multimédia interne Audiovisuel/Multimédia externe Hypermedia interactifs internes Hypermedia interactifs externes Événementiel interne Événementiel externe Réunions / Négociations Réseaux/Médiations extérieures Formation Liaison DRH

Liaisons agences de publicité Liaisons dir. marketing Com. sociale int. Com. de crise int. Com. de crise ext. Com. financière int. Com. financière ext. Groupe de pression Documentation Com. produit
Com. institutionnelle/Notoriété
Relations publiques/Presse
Mécénat/Parrainage
Stratégie de com externe
Stratégie interne
Analyse stratégique et globale
Recommandation stratégique
Conseil interne
Conseil externalisé
Concep./Gestion Plan de com 


\section{Les profils}

La modélisation proposée permet ensuite de dresser facilement le profil type, soit de l'individu évalué (ou auto-évalué...), soit d'une formation au complet (par moyenne de tous les profils types) ou encore d'un ensemble de tâches à assumer.

106 En voici quelques exemples très rapidement commentés à partir de profils types de métiers :

1. Le « dircom idéal »

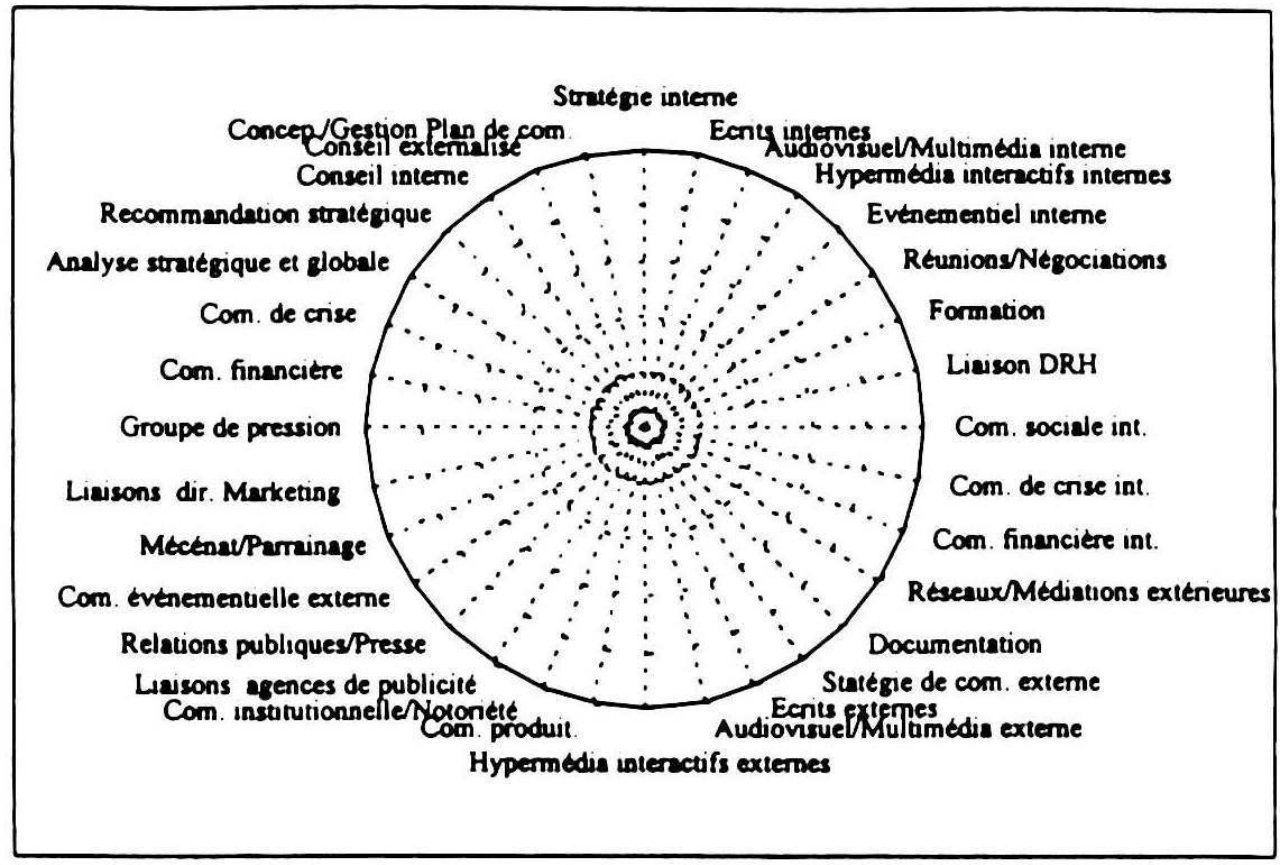

Son profil devrait être évidemment le cercle parfait? Il serait excellent partout, aussi bien en pratique (s'il est le "patron", il peut remplacer n'importe lequel de ses collaborateurs?) qu'en théorie. Naturellement, cet idéal n'est qu'une vue de l'esprit, voici quelques exemples plus réels et concrets. 


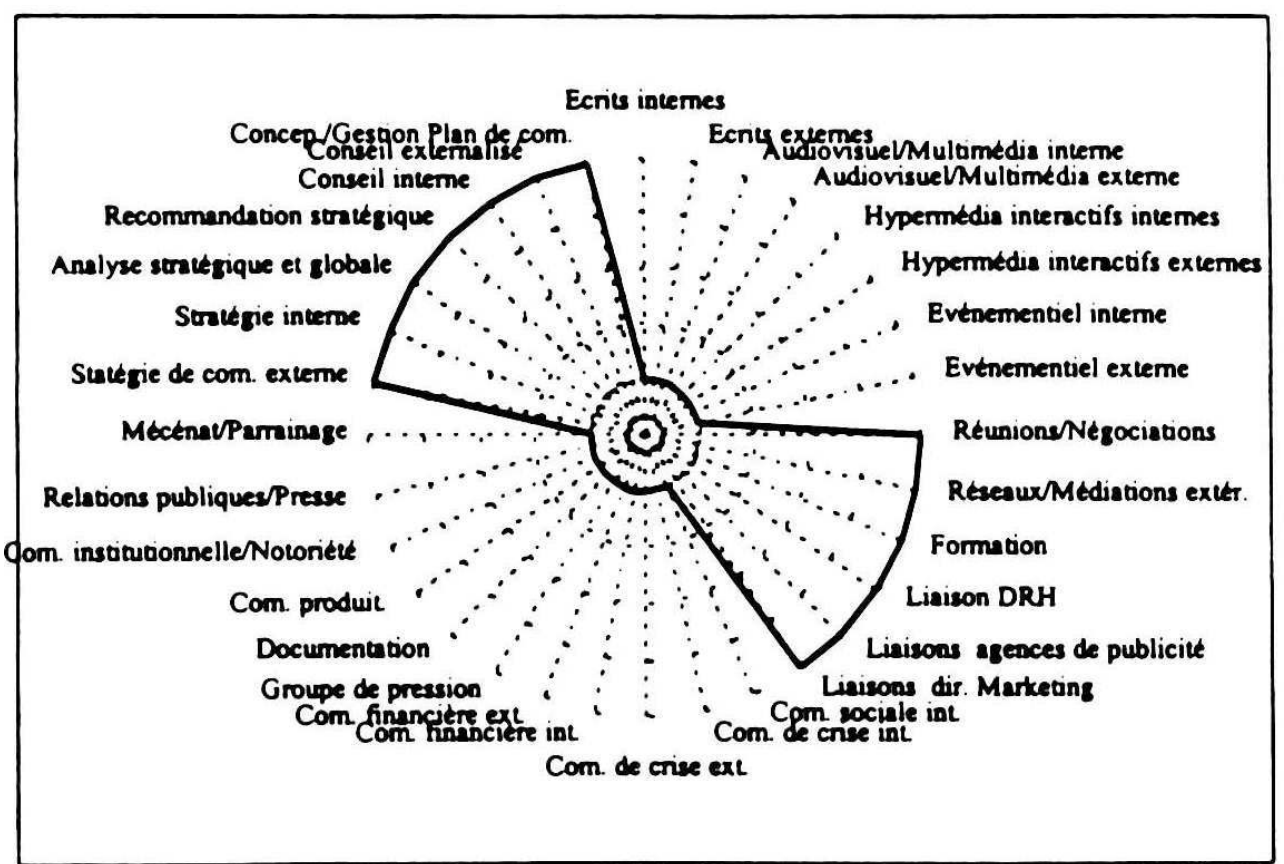

Le graphique en forme de nœud papillon (caricature de la fonction?) montre une symétrie entre les compétences directement stratégiques et celles liées à l'appartenance et à l'activation de réseaux.

3. Le stratège en communication externe

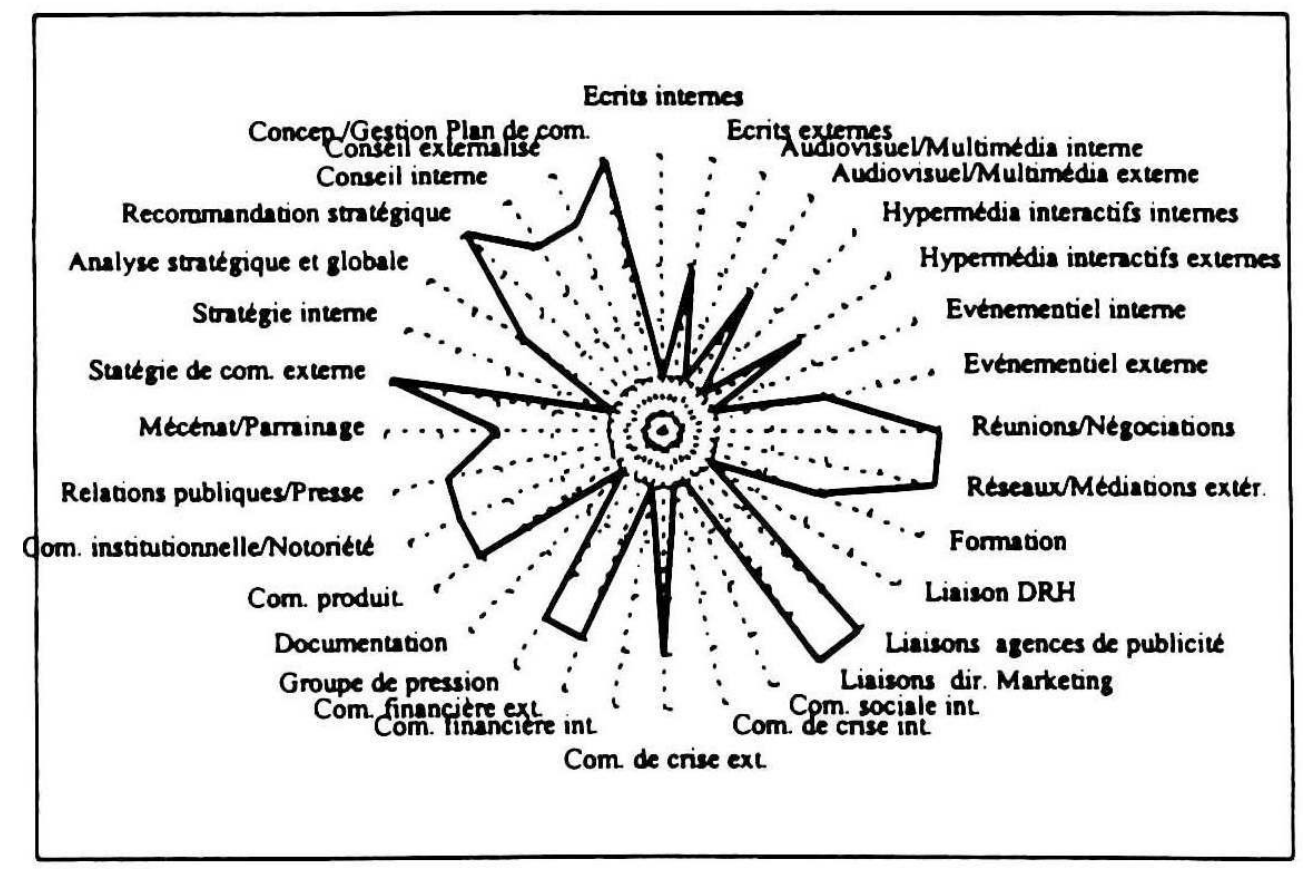


4. Le stratège en communication interne

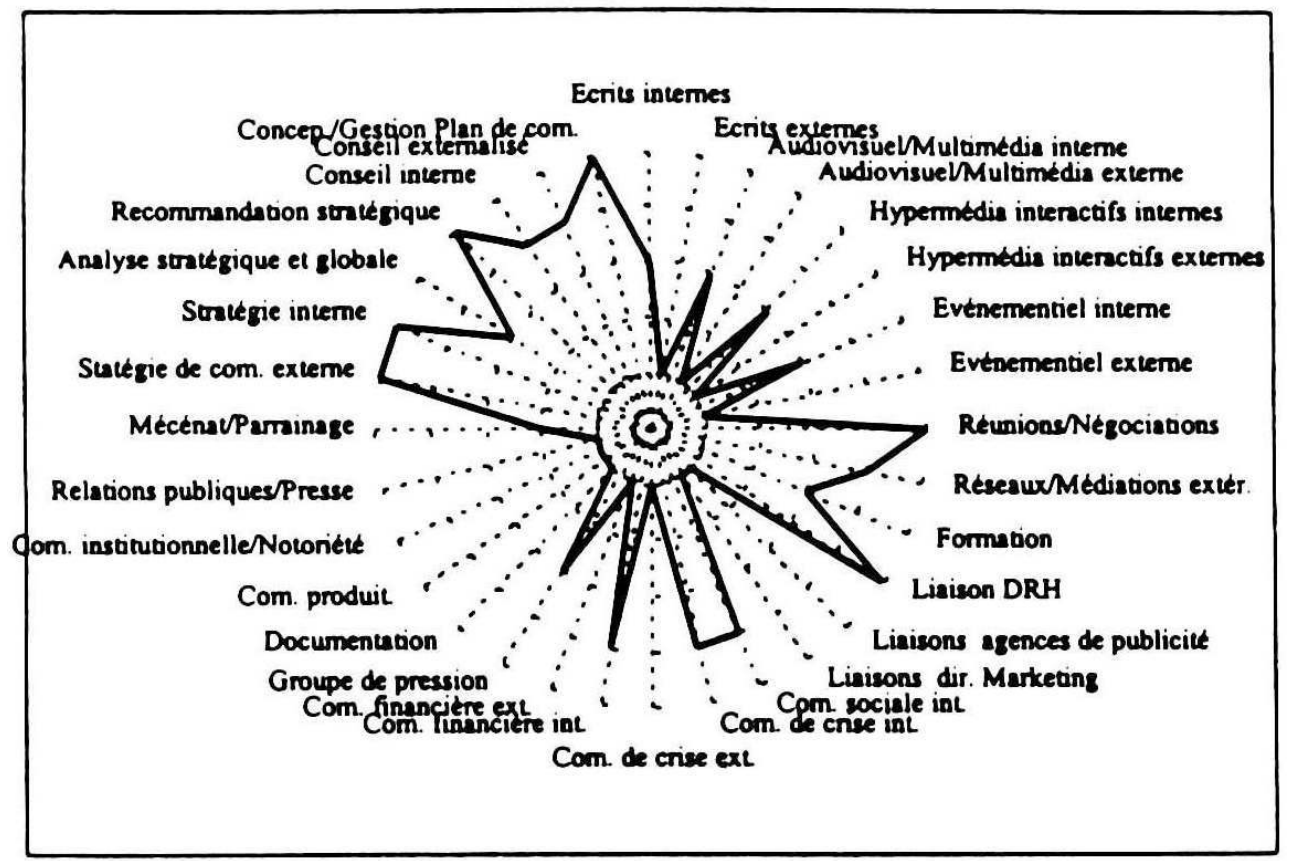

5. Le technicien multimédia (ou l'infographiste)

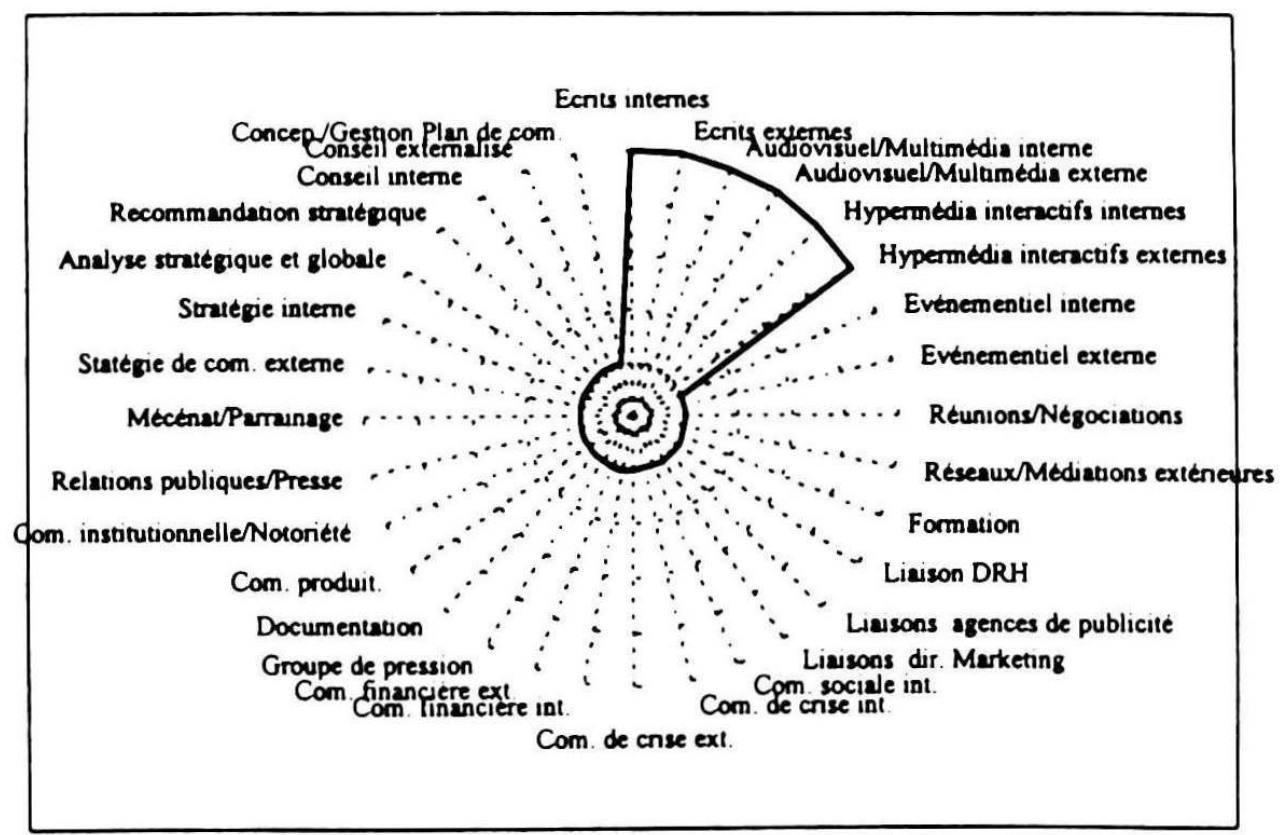




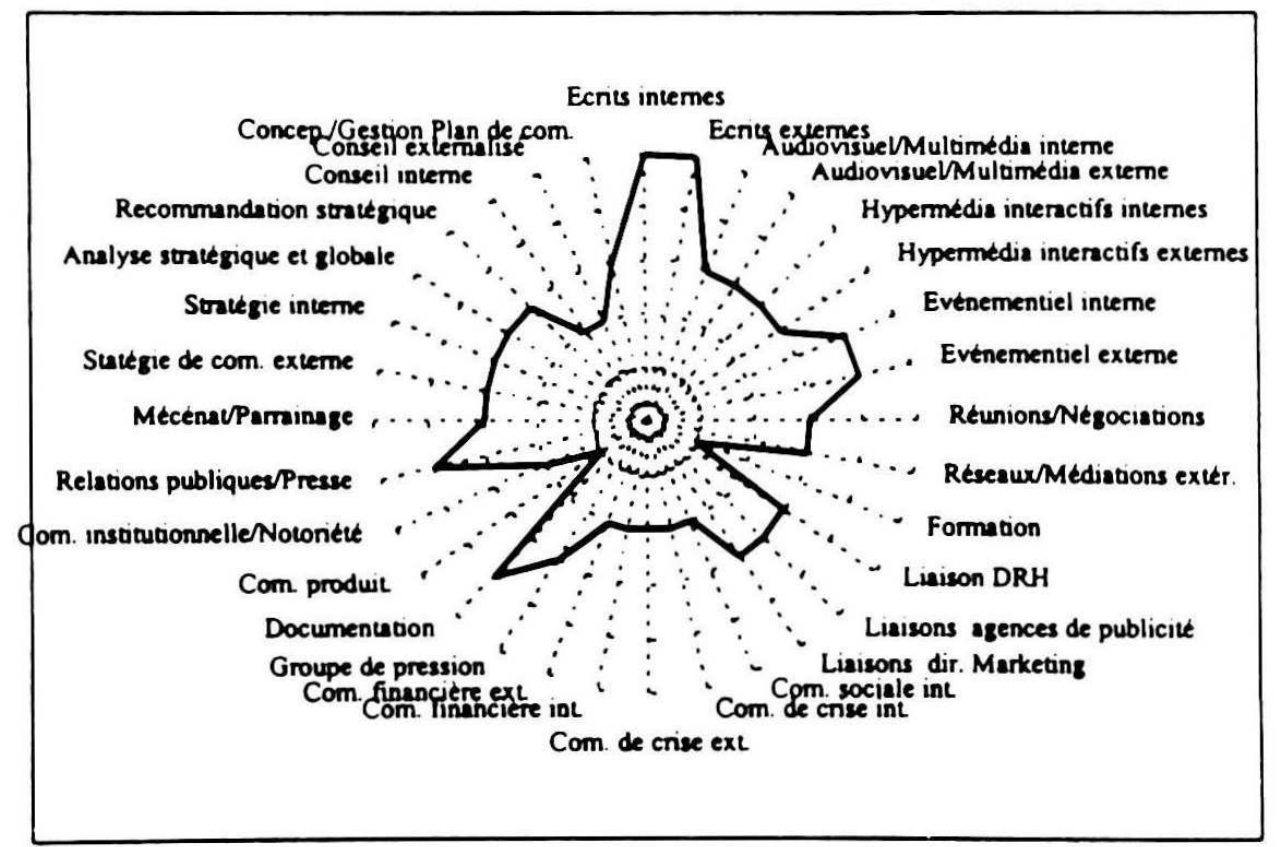

7. Le journaliste

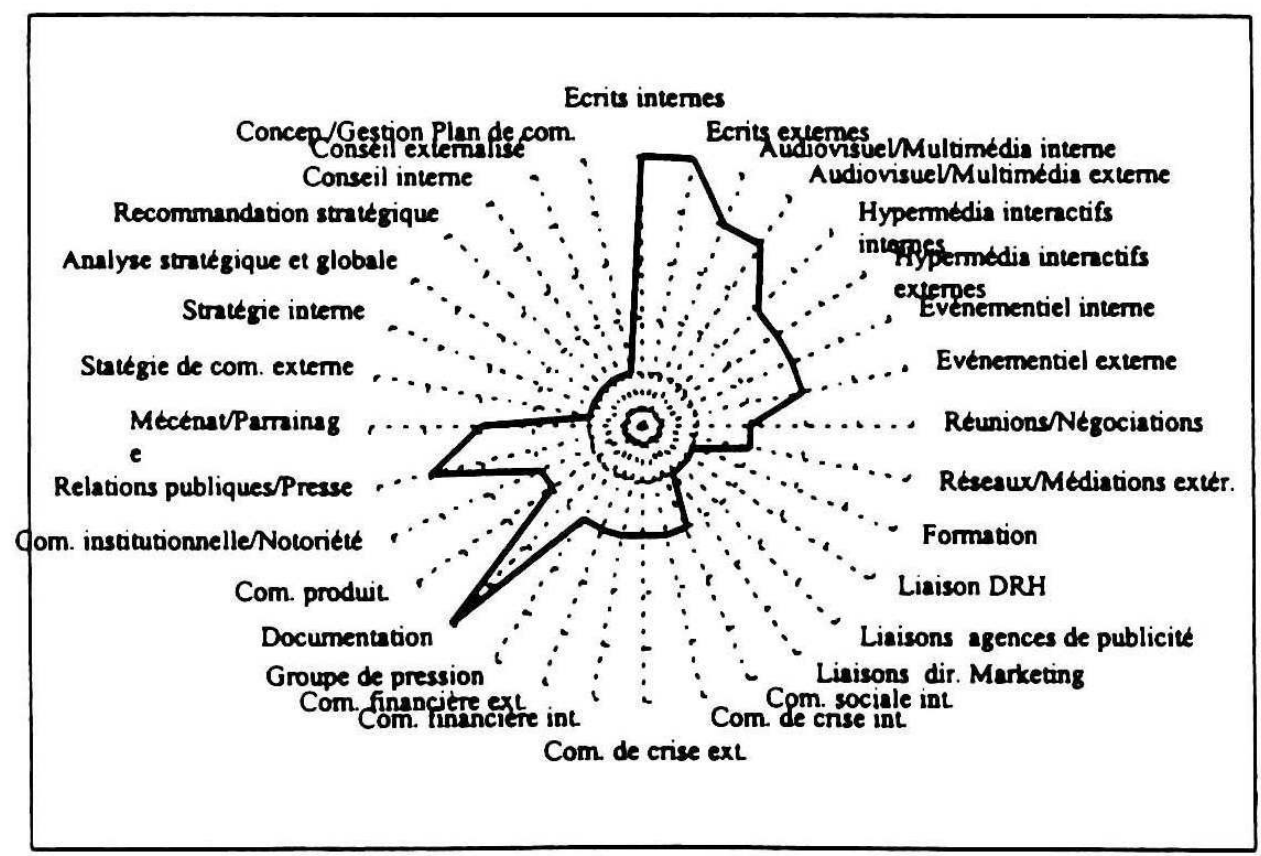

\section{Les métiers}

Comme on l'a vu, la grille de 139 descripteurs des activités de communication génère une trentaine de fonctions principales, cumulables ou non autour de l'externe ou de l'interne. Il est possible de déterminer facilement le profil type d'un individu, voire de l'évaluer pour examiner s'il correspond à un profil demandé. À titre d'exemple, la grille nous sert 
aussi chaque année à évaluer la formation à la communication d'entreprise : au moindre "trou » dans le polygone des fonctions, on repère un "manque» dans le cursus qu'il suffit ensuite de combler par un module approprié. Le dernier étage de cette combinatoire dynamique est donné par une liste d'une quinzaine de métiers de la communication d'entreprise et d'organisation. En voici l'état actuel, en évolution permanente :

Directeur de la communication (Responsable communication) Attaché (chargé, assistant) de communication interne Responsable (chargé) de la communication externe

Attaché de presse

Chargé de relations publiques

Journaliste d'entreprise (interne/externe)

Rédacteur en chef (interne/externe)

Concepteur/réalisateur audiovisuel (vidéo)

Concepteur télématique

Concepteur multimédia - hypermédia

Infographiste

Technicien PAO - maquettiste Concepteur rédacteur publicitaire Chef de projet (Directeur de création) Documentaliste (Chef documentaliste) Conseil en stratégie de communication (audit)

On notera que ne figurent que des métiers «reconnus", soit par les organismes spécialisés ou les associations professionnelles, soit l'intitulé des feuilles de paie; nous n'avons pas indiqué les «nouveaux métiers » non encore établis signalés au début de cet article. La diversité de ces métiers ne peut que confirmer la conclusion de l'enquête 1997 de l'UDA : pérennité assurée et professionnalisme de plus en plus grand. Si les formations sont à la hauteur, les diplômés de communication d'entreprise peuvent nourrir de bons espoirs d'insertion et de carrière...

\section{Conclusion : un secteur professionnel en plein essor}

121 Le plus grand risque pour les diplômes de communication d'entreprise serait certainement une inflation de diplômés car la situation actuelle limitant fortement les flux (BTS, IUT, MST, IUP, DESS...) est favorable à l'emploi. Avec le référentiel des fonctions dont un aperçu de la modélisation a été présenté dans cet article, les entreprises disposent d'un outil pour définir quel profil correspond à tels types de tâches; les responsables de formation peuvent combiner entre eux divers descripteurs pour examiner leur cohérence par rapport à un métier, les étudiants peuvent s'en servir pour mieux se connaître ou s'auto-évaluer et à terme trouver plus facilement des emplois correspondant à leurs aspirations et compétences. 


\section{NOTES}

1. Voir les enquêtes bisannuelles de l'UDA (Union des Annonceurs) "Responsables de communication, qui êtes vous?» et diverses autres études réalisées par des associations professionnelles. On peut aussi se tourner vers l'APEC qui découvre les métiers de la communication depuis peu de temps

2. À titre d'exemple, l'annuaire de la maîtrise de sciences et techniques de «Presse et communication d'entreprise » de l'université Jean Monnet (St. Etienne) fait état d'environ $80 \%$ d'anciens étudiants ayant un emploi en communication d'entreprise.

3. La dernière enquête de l'UDA, déjà citée, indique que pour la première fois en 1997, les recrutements ont été majoritairement effectués parmi des diplômés en communication, ce qui est loin d'être le cas dans d'autres secteurs, par exemple en journalisme

4. Que nos amis du secteur de l'information se rassurent, celle ci est évidemment omniprésente dans la communication, c'est par simplification (ou abus?) de langage que nous recourons au vocable «communication » sans mésestimer ce qu'il contient légitimement d'information, au sens de la métaphore de Robert Escarpit entre le carburant et le moteur...

5. Sur les directeurs de la communication, voir essentiellement l'ouvrage de Jacques Walter, Directeurs de la communication. Les avatars d un modèle professionnel. Paris, L'Harmattan, 1995

6. La notion de «métier» employée ici est bien évidemment celle des nomenclatures officielles, par exemple celles du ROME et des approches les plus modernes (nombre de «métiers" représentés dans une entreprise).

7. Voir évidemment les nombreux articles consacrés par la présente revue a ces questions

8. Par manque de place, nous ne donnerons pas ici la combinatoire employée, elle est classique et correspond à ce que tout connaisseur de la communication des organisations pourrait déterminer.

\section{RÉSUMÉS}

En se pérennisant les tâches dédiées à la communication se diversifient et se complexifient. Pour dresser un panorama suffisamment clair des métiers (une quinzaine), il faut en amont construire une grille des fonctions de base (139) et des grandes tâches (32). Avec une modélisation qui les combine de manière dynamique, il devient possible de clarifier les profils, les niveaux de compétences et de contribuer à la réflexion prospective.

While becoming perennial, communication-dedicated tasks become more diverse and complex. In order to have a clear overview of the trades (about 15 of them), a grid featuring the basic functions (139) and the most relevant tasks (32) must be implemented beforehand. Using modelling which combines them dynamically, it becomes possible to sort out and highlight the various profiles, skill levels, hence contributing to a prospective consideration on communication trades. 
INDEX

Mots-clés : métiers de la communication, fonctions, profils, compétences

\section{AUTEUR}

\section{JEAN-LUC MICHEL}

Jean-Luc Michel, docteur de l'Université Paris 7, Professeur, et directeur du département de communication de l'Université Jean Monnet à Saint-Étienne. Il est auteur de La Distanciation, Essai sur la société médiatique (L'Harmattan) et Le Mémoire de recherche (Ellipses). 